\title{
DYNAMICS OF PHOTOINDUCED ELECTRON TRANSFER IN MULTIPORPHYRIN NANOASSEMBLIES
}

\author{
D. KILIN \\ University of South Dakota, Vermillion SD 57069, USA
}

E. ZENKEVICH

Belarusian National Technical University, 220013 Minsk, Belarus

C. VON BORCZYSKOWSKI

Chemnitz University of Technology, NANOMA, 09107 Chemnitz, Germany

\begin{abstract}
In self-assembled nanoscale porphyrin triads based on $\mathrm{Zn}$-octaethylporphyrin chemical dimer (donor, D) and dipyridyl substituted porphyrin free base (acceptor, A), fluorescence quenching of $\mathrm{D}$ (down to 1.7-10 ps) and A (by 1.3-1.6 times) subunits is strongly dependent on the solvent polarity (toluene-acetone mixtures) and temperature $(77-350 \mathrm{~K})$. The obtained experimental findings are analyzed using the reduced density matrix formalism in the frame of Haken-Strobl-Reineker approach taking into account the energy transfer, charge separation, and the dephasing of coherence between the excited electronic states of the triad.
\end{abstract}

\section{Introduction}

At the moment, there are many promising ways in which principles of natural elementary photoprocesses (e.g. phosynthesis, vision, etc.) are being applied to the engineering and preparation of various man-made supramolecular nanodevices in the nascent field of molecular electronics that function as photoinduced molecular switches and nanowires, photonic wires, optoelectronic gates, and multimolecular architectures for information storage [1,2]. Spontaneous self-assembly, which occurs as a result of the complementarity of superstructure components, has enabled the preparation of molecules, macromolecules, and supramolecules of nanoscale dimensions, as well as of mechanically interlocked architectures [3].

Recently, we have shown that in nanoscale self-assembled porphyrin triads (Fig. 1) based on Zn-octaethylporphyrin chemical dimer (donor, D) and ligand as dipyridyl substituted porphyrin free base (acceptor, A), fluorescence quenching of $\mathrm{D}$ (down to $1.7-10 \mathrm{ps}$ ) and A (by $\sim 1.3-1.6$ times) subunits is strongly dependent on the solvent polarity (toluene-acetone mixtures) and temperature (77-350 K) [4]. In this contribution, we present theoretical analysis of the experimental data on the non-radiative relaxation processes in porphyrin triads 
(including energy transfer, charge separation, and the dephasing of coherence between the excited electronic states), using the reduced density matrix approach based on the Haken-Strobl-Reineker formalism with neglection of the vibrational substructure of the electronic states [5]. We show that this approach provides both qualitative and quantitative description of time-resolved and steady-state properties such as fluorescence quenching for both subunits of the triad and gives a good agreement with performed experiments.

\section{Results and discussion}

Herein, we discuss the peculiarities of photoinduced electron transfer (PET) processes competing with the Förster energy transfer (FRET) in porphyrin triads on the basis of the experimental findings and theoretical calculations. Multiporphyrin nanoassemblies are formed via coordination interactions of Zn-octaethylporphyrin chemical dimer (ZnPD) as energy and electron donor (D) and dipyridyl-substituted free base porphyrin $\left(\mathrm{H}_{2} \mathrm{P}\right)$ as the acceptor (A), see Fig. 1.
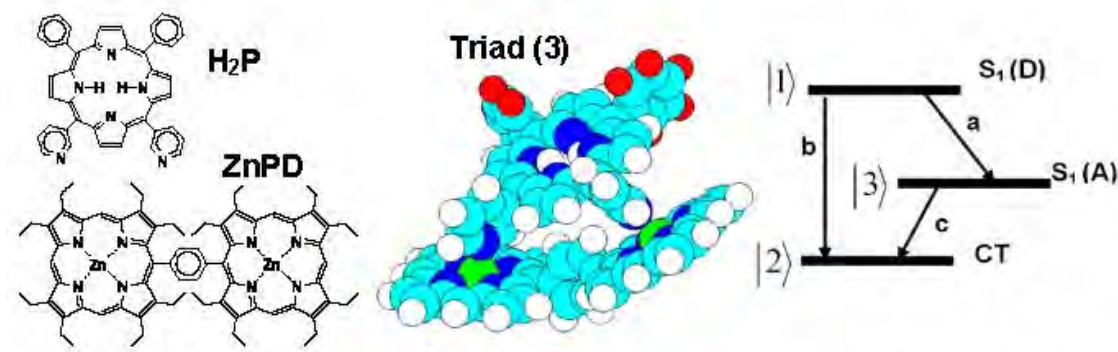

Figure 1. Chemical structure of Zn-octaethylporphyrin chemical dimer ZnPD (1), dipyridyl substituted porphyrin free base $\mathrm{H}_{2} \mathrm{P}$ (2, ligand), optimized (HyperChem, release 4.0, PM3) structure of porphyrin triad (3), as well as scheme of excited states $S_{1}(D), S_{1}(A), C T$ and photoinduced relaxation processes in the triad: (a) weak dissipative Foerster energy transfer, (b) sequential electron transfer, (c) weak coherent and dissipative charge transfer. The decay of charge-transfer

CT-state (charge recombination to low-lying excited triplet state) is slow and is not shown.

It was found that the experimentally observed relaxation of excited states $\mathrm{S}_{1}(\mathrm{D}), \mathrm{S}_{1}(\mathrm{~A})$ and $\mathrm{CT}$ do not show any dynamic effects within $120 \mathrm{fs}-1.4 \mathrm{ps}$ associated with vibrational substructure. It means that the vibrational relaxation is much faster than PET. The influence of the quantum bath on a single excitation has been taken into account (represented by a stochastic potential which is Gaussian, Markovian and $\delta$-correlated in time). In this case, the coherent energy transport may be described by the transition matrix elements between radicals of the porphyrin triad. Thus, the dynamics of the excited states 
$|1\rangle=\left|\mathrm{ZnPD}^{*}-\mathrm{H}_{2} \mathrm{P}\right\rangle, \quad|2\rangle=\left|\mathrm{ZnPD}^{+}-\mathrm{H}_{2} \mathrm{P}^{-}\right\rangle$, and $|3\rangle=\left|\mathrm{ZnPD}-\mathrm{H}_{2} \mathrm{P}^{*}\right\rangle$ is described by the equation of motion for the relevant reduced density matrix with neglecting the vibrational substructure of the electronic states

$$
\begin{aligned}
\frac{\partial}{\partial t} \sigma_{\kappa \lambda}= & -\frac{i}{\hbar}\left(\left[H_{S}, \sigma\right]\right)_{\kappa \lambda}+2 \delta_{\kappa \lambda}\left\{\Gamma_{\mu \kappa}\left[n\left(\omega_{\mu \kappa}\right)+1\right]+\Gamma_{k \mu} n\left(\omega_{k \mu}\right)\right\} \sigma_{\mu \mu} \\
& -\Sigma_{\mu}\left\{\Gamma_{\mu \kappa}\left[n\left(\omega_{\mu \kappa}\right)+1\right]+\Gamma_{k \mu} n\left(\omega_{k \mu}\right)+\Gamma_{\mu \lambda}\left[n\left(\omega_{\mu \lambda}\right)+1\right]+\Gamma_{\lambda \mu} n\left(\omega_{\lambda \mu}\right)\right\} \sigma_{\kappa \lambda} \\
& +\left\{\Gamma_{\lambda \kappa}\left[2 n\left(\omega_{\lambda \kappa}\right)+1\right]+\Gamma_{\kappa \lambda}\left[2 n\left(\omega_{\kappa \lambda}\right)+1\right]\right\} \sigma_{\lambda \kappa} .
\end{aligned}
$$

Here the triad Hamiltonian $H_{S}$ includes the energies $E_{\lambda}$ of the corresponding states and couplings between them, $n(\omega)=\left[\exp \left(\hbar \omega / k_{\mathrm{B}} T\right)-1\right]^{-1}$ denotes Bose-Einstein distribution, $\Gamma_{\kappa \lambda}$ is the damping constant, and $\kappa, \lambda, \mu=1,2,3$. The energies $E_{1}=2.1 \mathrm{eV}$ and $E_{3}=1.91 \mathrm{eV}$ are taken from [3,4]. The energy of state $|2\rangle$ depending on the solvent polarity can be calculated using so-called Weller formula

$$
E_{2}(\varepsilon)=E_{2}\left(\varepsilon_{t}\right)+\left(\frac{1}{\varepsilon}-\frac{1}{\varepsilon_{t}}\right) \frac{e^{2}}{4 \pi \varepsilon_{0}}\left(\frac{1}{2 r_{D}}+\frac{1}{2 r_{A}}-\frac{1}{r_{D A}}\right) \text { with } \varepsilon_{e f f}=\varepsilon_{t}+c \frac{3\left(\varepsilon_{a}-\varepsilon_{t}\right) \varepsilon_{t}}{\varepsilon_{a}+2 \varepsilon_{t}} .
$$

Experimental solvent mixture consists of toluene and a small concentration $\mathrm{c}$ of polar acetone characterized by the static dielectric constants $\varepsilon_{t}=2.38$ and $\varepsilon_{a}=10$, correspondingly. In this case, the effective dielectric constant $\varepsilon_{\text {eff }}$ of the solvent mixture may be calculated as follows.

To describe the energy relaxation in the triad physically reasonable values of the model parameters, such as coherent and dissipative couplings and energy of states were calculated as well as reasonable values of relaxation constants

\begin{tabular}{|c|c|c|c|}
\hline Coupling & $\begin{array}{l}\text { Value, } \\
\text { meV }\end{array}$ & Physical Process & Comment \\
\hline$v_{12}$ & $\leq 60$ & electron transfer $\mathrm{D}^{*} \mathrm{~A} \rightarrow \mathrm{D}^{+} \mathrm{A}^{-}$ & Induced by the wave function overlap \\
\hline$v_{32}$ & 3 & hole transfer $\mathrm{DA}^{*} \rightarrow \mathrm{D}^{+} \mathrm{A}^{-}$ & $\begin{array}{l}\text { Weakened by the screening field of } \\
\text { the electron from the LUMO of the } \\
\text { acceptor }\end{array}$ \\
\hline$v_{13}$ & 12 & energy transfer $\mathrm{D}^{*} \mathrm{~A} \rightarrow \mathrm{DA}^{*}$ & $\begin{array}{l}\text { Induced by the dipole-dipole } \\
\text { interaction of the excited states } \\
v_{13} \propto p_{\mathrm{D}^{*} \mathrm{~A}} p_{\mathrm{DA}^{*}} / r_{\mathrm{DA}}^{3}\end{array}$ \\
\hline$\Gamma_{12}$ & 0.41 & loss of coherence for $\mathrm{D}^{*} \mathrm{~A} \rightarrow \mathrm{D}^{+} \mathrm{A}^{-}$ & $\begin{array}{l}\text { Interaction of the transition dipole } \\
\text { with environmental dipoles }\end{array}$ \\
\hline$\Gamma_{32}$ & 2.50 & loss of coherence for $\mathrm{DA}^{*} \rightarrow \mathrm{D}^{+} \mathrm{A}^{-}$ & $\begin{array}{l}\text { Induced by the interaction with the } \\
\text { environment }\end{array}$ \\
\hline$\Gamma_{13}$ & 0.37 & loss of coherence for $\mathrm{DA}^{*} \rightarrow \mathrm{DA}^{*}$ & $\begin{array}{l}\text { Estimated by taking into account } \\
\text { other channels of dissipation } \\
\gamma_{i j}=\sum_{k}\left(d_{i k}+d_{k i}\right)\end{array}$ \\
\hline
\end{tabular}
were also estimated using the width of the relevant bands in the absorption spectra of the triad. The values of the couplings are collected in Table 1 .

Table 1. Coupling values for the processes under study. 
For each parameter set we calculated the relevant reduced density matrix $\sigma_{\kappa \lambda}(t)$ numerically. At $t=\infty$ the diagonal elements of density matrix arrive to the quasiequilibrium values. These values correspond to the fluorescence intensity of the triad subunits and have been compared with experimental data as shown in Fig. 2.
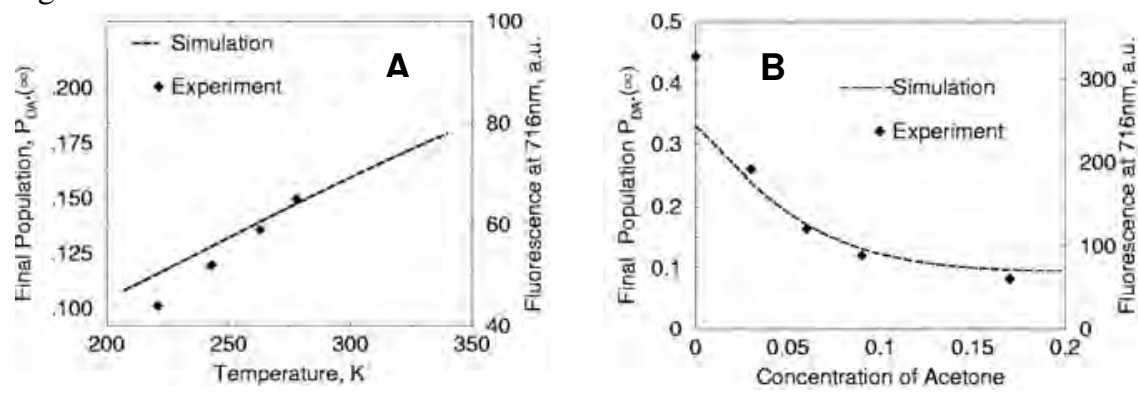

Figure 2. Temperature (A) and solvent polarity at $293 \mathrm{~K}$ (B) dependence of the fluorescence quenching for porphyrin extra-ligand $\mathrm{H}_{2} \mathrm{P}$ in the triad in toluene-acetone mixture.

Calculated dependencies of the acceptor population in the $S_{1}$-state reflecting the dependence of the porphyrin extra-ligand fluorescence intensity on temperature and solvent polarity in the triad are in a reasonable accordance with experimental data. In addition, the theoretical analysis predicts that in the presence of polar admixture (5-15 vol.\% of acetone) the increase of temperature induces the crossover from the coherent to the incoherent type of the quantum particle transport in nanoscale self-assembled multiporphyrin complexes.

\section{Acknowledgments}

Financial support by Belarussian SPSR "Convergence 3.2.08" is gratefully acknowledged.

\section{References}

1. J.-H. Fuhrhop, Langmuir. 30, 1 (2014).

2. Y. Terazono, G. Kodis, M. Chachisvilis, B. R. Cherry, M. Fournier, A. Moore, T. A. Moore, D. Gust, J. Am. Chem. Soc. 12, DOI: $10.1021 /$ ja510267c (2014).

3. E. I. Zenkevich, C. von Borczyskowski, J. Porphyrins Phthalocyanines. 18, 1 (2014).

4. E. I. Zenkevich, C. von Borczyskowski. in: Multiporphyrin Arrays: Fundamentals and Applications (Pan Stanford Publishing Co. Pte. Ltd.: Singapore), 5, p. 217-288 (2012).

5. M. Schreiber, D. Kilin, U. Kleinekathoefer, J. Lumin. 83-84, 235 (1999). 